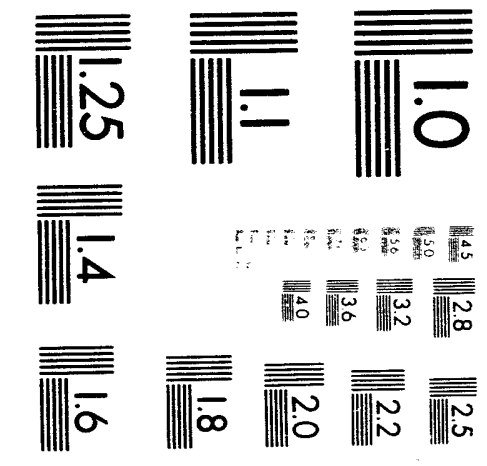



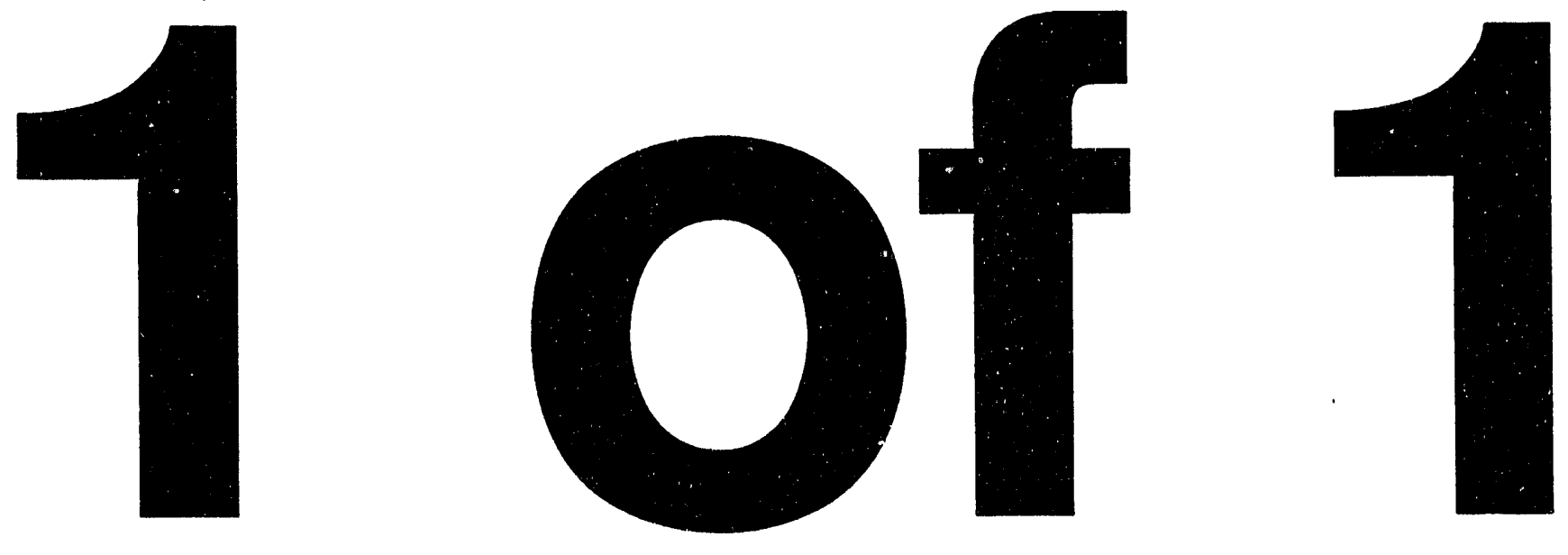


\section{New Equation of State for Stainless Steel 347 \\ J. C. Boettger}




\title{
New Equation Of State For Stainless Steel 347
}

by

J. C. Boettger

\begin{abstract}
A new SESAME equation of state (EOS) for stainless steel 347 has been generated using the computer program GRIZZLY, and has been added to the SESAME EOS library as material number 4271 . This new EOS is superior to its predecesser (material number 427() ) in several respects.
\end{abstract}

\section{INTRODUCTION}

The SESAME equation of state (EOS) library has been an important tool for scientists throughout the world since its inception in $1971 .^{1}$ During this time, the numerical techniques used to calculate the phenomenological EOSs contained in SESAME have heen significantly improved. Perhaps the single most important advance to date :va:; the development of the computer program GRIZZLY as a general ptrpos of te fo.

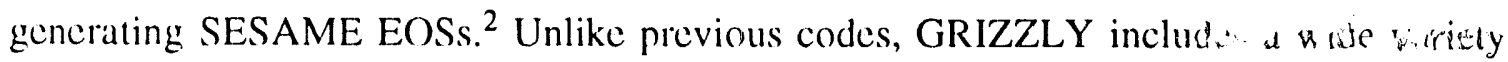
of models for calculating all of the necessary components of a SESAire r: 8 .

Given the long history of the SESAME library, it is to be expect i it" : inrin. if the older EOSs in the library (particularly those developed prior to GRIZZ1 , would not he of a quality consistent with the current state of the art and should be updated as 
the need arises. One such EOS is that lor stainless stect; material number 4270. Unlike more recent additions to the SESAME library, there is no clear documentation of the methods used to generate material number 427(). In addition, the original EOS was subsequently partitioned into so-called two-temperature tables (individual ionic and electronic contributions) using approximations which are crude at hest.

In this investigation, a new EOS has heen constructed for stainless steel 347 using GRIZZLY. ${ }^{2}$ This work is a direct response to requests by users of the SESAME library. The new EOS has thermodynamically consistent two-temperature EOS tables and includes vapor pressure and melting tables. The new EOS has been added to the SESAME lihrary as material number 4271.

In the next section, the models and parameters used to generate the EOS for stainless steel are described in detail. This new SESAME EOS is then discussed and compared with experimental data in Section III.

\section{METHODOLOGY}

Most of the EOSs in the SESAME lihrary are partitioned into three terms for the pressure $P$, the internal energy $E$, and the Helmholtz free energy $A$ :

$$
\begin{aligned}
& P(\rho, T)=P_{s}(\rho)+P_{n}(\rho, T)+P_{\iota^{\prime}}(\rho, T) \\
& E(\rho, T)=E_{s}(\rho)+E_{n}(\rho, T)+E_{\iota^{\prime}}(\rho, T) \\
& A(\rho, T)=A_{s}(\rho)+A_{n}(\rho, T)+A_{\iota^{\prime}}(\rho, T)
\end{aligned}
$$

where $\rho$ is the density and $T$ is the temperature. (In the SESAME library, discrete values of $\rho$ and $T$ are chosen to form a mesh on which $P, E$, and $A$ are stored.) The 
subscripts $s, n$, and $e$ denote the contributions from the static lattice (i.e., frozen nuclei) cold curve (zero temperature isotherm), the nuclear motion, and the thermal electronic excitations, respectively. It is thus possible to calculate (or update) each term independently using any desired model. This partitioning also allows users to perform two-temperature calculations; i.e., calculations in which the ions and the electrons are allowed to have different temperatures. Here, all three components of the EOS for stainless steel have been generated with GRIZZLY. ${ }^{2}$

In GRIZZLY, the only model currently available for calculating the thermal elecIronic contributions is the Thomas-Fermi-Dirac (TFD) model. ${ }^{3}$ First, thermal electronic contributions were generated for each individual type of atom in stainless steel 347. Those monatomic thermal electronic EOSs were then combined via additive volume mixing ${ }^{2}$ to form the total electronic contribution to the EOS. This portion of the calculation requires an ambient density $\left(\rho_{0}\right)$ for the total EOS and the weight fraction $\left(W_{i}\right)$, atomic number $\left(Z_{i}\right)$, and atomic mass $\left(A_{i}\right)$ for each species of atom $(i)$. Stainless steel 347 is a well-defined type of steel with an ambient density of about $7.91 \mathrm{gm} / \mathrm{cc}^{4}$ and is composed of Fe combined with $17 \%$ to $19 \% \mathrm{Cr}$, $9 \%$ to $13 \% \mathrm{Ni}$, less than $2 \% \mathrm{Mn}$, less than $1 \%$ Si, and traces of several other elements. ${ }^{5}$ For material number 4271 , stainless sted 347 has heen approximated hy a mixture of $7(1 \%) \mathrm{Fe}, 19 \% \mathrm{Cr}$, and $11 \% \mathrm{Ni}$; the same composition as was used for material number 4270 . The atomic masses used for $\mathrm{Fe}, \mathrm{Cr}$, and $\mathrm{Ni}$ were $55.85,52 .()()$, and 58.71 , respectively. ${ }^{6}$

The thermal nuclear contributions for material number 4271 were obtained with the recently developed JDJNUC nuclear model ${ }^{7}$ in GRIZZLY. In this model, the 
material is treated as a Dehye solid at low temperatures and as an ideal gas at high temperatures. The JDJNUC model smoothly switches hetween these two limits for temperatures near the melt line, which is obtained from the Lindemann law. At the melting point on each isotherm, the JDJNUC model builds in a small two-phase region to simulate the melting transition. This part of the calculation requires an ambient value for the melt temperature $\left(T_{m}\right)$ and some analytical form for the Gruncisen parameter as a function of density $[\gamma(\rho)]$. The Debye temperature $\left(\Theta_{D}\right)$ is then computed internally from $T_{m}$ using an empirical relationship based on the behavior of pure elements. Here, the melt temperature of stainless steel 347 was approximated by that of elemental Fe; $T_{m}=18\left(18 \mathrm{~K}^{6}\right.$

A generalized version of the standard CHART-D model ${ }^{8}$ has heen used to describe $\gamma(\rho)$; the IGRUN=7 option in GRIZZLY. ${ }^{2}$ In this formulation, the Gruneisen parameter is expressed as a quadratic function of $\rho$ in the expanded region and as a quadratic function of $1 / \rho$ in the compressed region. Four items of input are required to completely specify $\gamma(\rho)$; ambient values of $\gamma$ and its derivative with respect to $\ln \rho$ (GAMREF and DGAMMA), and its asymptotic values $\gamma(0)$ and $\gamma(\infty)$. For $\gamma(())$ and $\gamma(\infty)$ the default values of 1 and $2 / 3$ were used. GAMREF was taken to be the ambient Gruneisen parameter for Fe; i.e., 1.66." Finally, DGAMMA was chosen to have a small negative value (-().(1)5) to ensure that the nuclear isotherms are smooth.

At modest compressions $(\leq 2.1)$, the static lattice cold curve for material number 4271 was calculated hy removing thermal contrihutions from an input Hugoniot expressed as a straight line fit to experimental shock velocity $\left(U_{s}\right)$ vs. particle velocity 
$\left(U_{p}\right)$ data. $^{4.10}$ The input Hugoniot used here was $U_{s}=4.62+1.42 U_{p}$, in $\mathrm{km} / \mathrm{s}^{4}$ For high compressions, the cold curve was required to smoothly extrapolate to a mixed TFD cold curve. This method of calculation ensures that the compressed EOS will reproduce the experimental Hugoniot and also have the correct asymptotic behavior. In the expanded region $\left(\rho<\rho_{0}\right)$, the cold curve was fored to have a generalized Lennard-Jones form ${ }^{2}$ that was constrained to smoothly connect with the compressed portion of the eold curve and have the correct cohesive energy $\left(E_{c}\right)$. The cohesive energy of stainless steel has heen estimated by that of elemental Fe, $99.4 \mathrm{kcal} / \mathrm{mole}{ }^{9}$ The only remaining parameter needed for the calculation is a parameter (FACLJ) which controls the shape of the cold curve in the expanded region. Here, FACLJ has been used to adjust the output values of the critical temperature $\left(T_{c}\right)$ and critical pressure $\left(P_{c}\right)$. It was found that reasonahle values for $T_{c}$ and $P_{c}$ could be obtained with FACLI $=() .7 ;$ sec helow.

\section{RESULTS}

Figure 1 shows a selected group of $P$ vs. $\rho$ isotherms from the new SESAME EOS for stainless sted 347. In general, the isotherms for material number 4271 do not exhibit any questionahle hehavior. It is also apparant that the new EOS for stainless stecl has a critical temperature of about $870(0 \mathrm{~K}$ and a critical pressure of about 19 khar. These values compare quite lavorahly with previous estimates which suggest $T_{c} \approx 9()(0) \rightarrow 10(0)() \mathrm{K}$ and $P_{c}=10$ khar. ${ }^{11}$ It is not possible to get a very accurate estimate of the critical point for material number 4270, hut simple inspection of that 
E(OS suggests a $T_{c}$ in the range of $8(0)() \rightarrow 1(0)()() \mathrm{K}$ and a $P_{c}$ which is in the range of $20 \rightarrow$ 30 khar. While these values are eomparable to those for 4271 , the newer EOS should give a sightly better description of stainless steel near the critical point.

Figure 2 compares the theoretical Hugoniots for material numbers 4270 and 4271 with experimental Hugeniot data for stainless steel $347 .{ }^{4}$ While hoth of the theoretical Hugoniots are in hasic agreement with the data, the Hugoniot for the newer EOS (4271) is in somewhat better agreement with experiment for $U_{p}$ values greater than 2.) $\mathrm{km} / \mathrm{s}$. It is also clear from Fig. 2 that the new SESAME EOS for stainless sted 347 should be reliable for all applications involving shock loading.

In general, although the total EOSs for 427() and 4271 are similar, the newer EOS should he slightly more accurate both in the vapor dome region and along the principal Hugoniot. In addition, as was noted earlier, the two-temperature tables for the older EOS are very noisy and were generated from the total EOS using very crude approximations; whereas, for material 4271, the total EOS was obtained directly from the Iwo-temperature tables. For these reasons, material number 4271 should be preferred over 4270 for virtually all applications. 


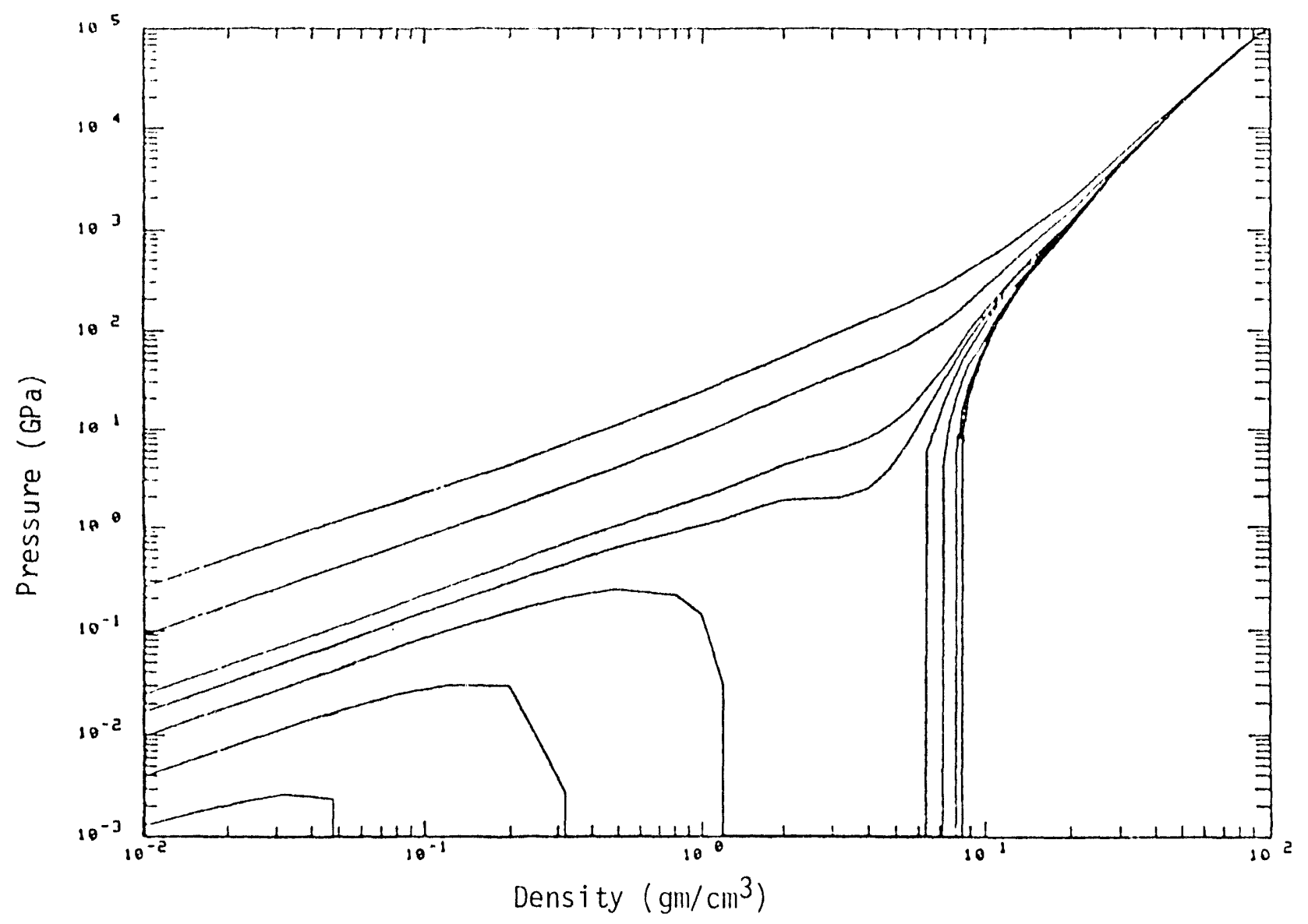

Figure 1. Selected isotherms from the new SESAME EOS for stainless steel 347. In order, the isotherms shown are for $T=0 \mathrm{~K}, 1160 \mathrm{~K}, 2900 \mathrm{~K}, 5800 \mathrm{~K}, 8700 \mathrm{~K}$, $116000 \mathrm{~K}, 29000 \mathrm{~K}$, and $58000 \mathrm{~K}$. 


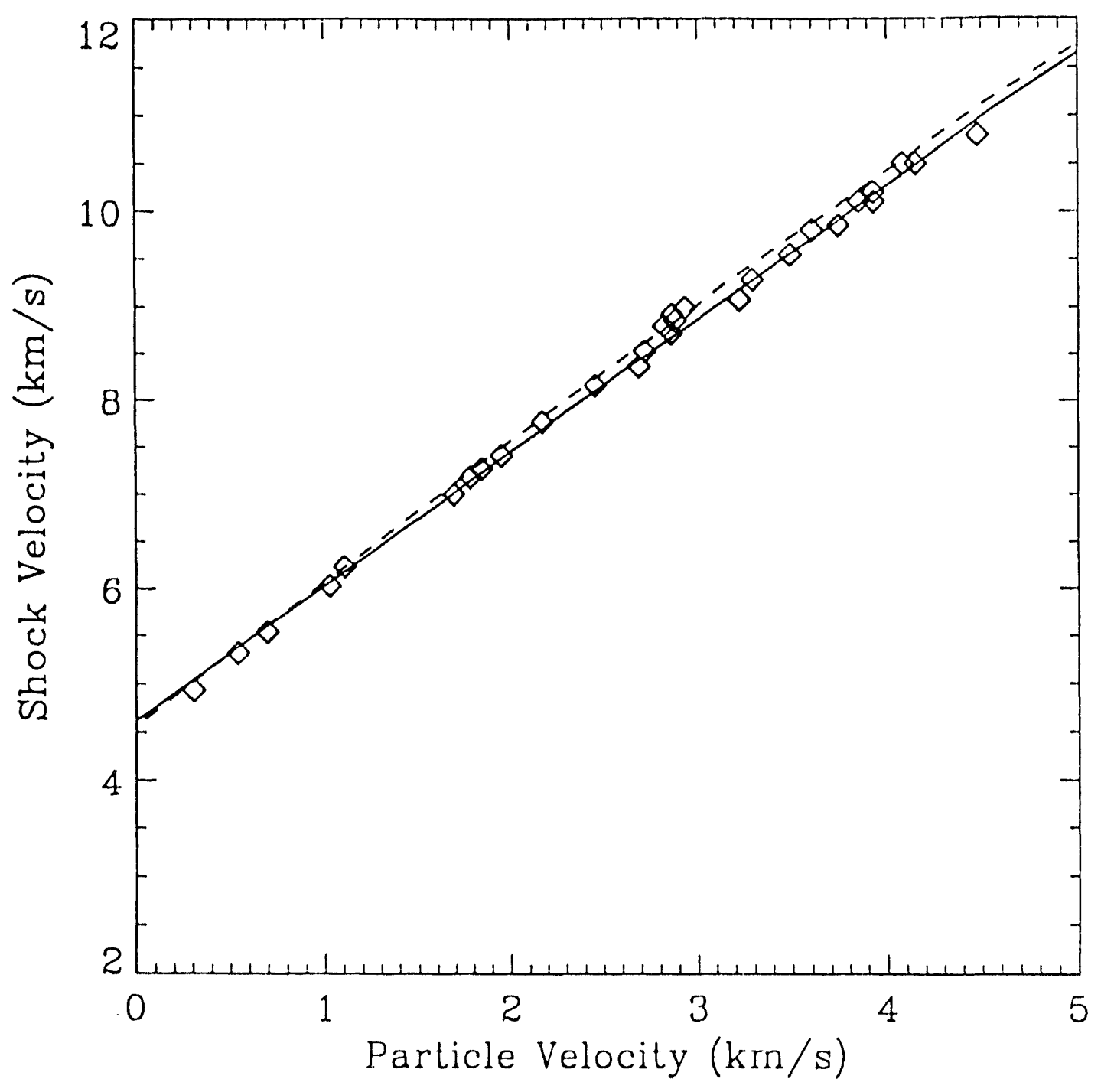

Figure 2. The theoretical Hugoniots for material numbers 4271 (solid line) and 4270 (dashed line) are compared with Hugoniot data for stainless steel 347. 


\section{REFERENCES}

1. K. S. Holian, editor, "T-4 Handhook of Material Properties Data Bases," Los Alamos National Laboratory Report LA-1016()-MS (November 1984).

2. Joseph Abdallah, Jr., "User's Manual for GRIZZLY," Los Alamos National Laboratory report LA-10244-M (September 1984).

3. R. D. Cowan and J. Ashkin, Physs. Rev. 105, 144 (1957).

4. S. P. Marsh, editor, LASL Shock Hugoniot Data (U. C. Press, Berkeley, CA, $198(1)$

5. R. C. Weast, editor, CRC Handhook of Chemistry and Physics, 69th Edition, (CRC Press, Inc., Boca Raton, FL, 1988).

6. N. W. Asheroft and N. D. Mermin, Solid State Physics (Holt, Rinchart, and Winston, New York, 1976).

7. J. D. Johnson, "A Generic Model For The Ionic Contribution To The Equation Of State," Los Alamos National Lahoratory Report LA-120)(3-MS (January 1991).

8. S. L. Thompson and H. S. Lawson, "Improvements to the CHART-D RadiationHydrodynamics Code III; Revised Analytic Equations of State," Sandia Laboratorices report SC-RR-71(1714 (1972).

9. K. A. Gschncidnci, Jr. Solid State Phys. 16, 276 (1964).

1). J. A. Morgan, High T. - High P. 7, 65 (1975).

11. R. W. Ohse and H. von Tippelskirch, High T. - High P. 9, 67 (1977). 

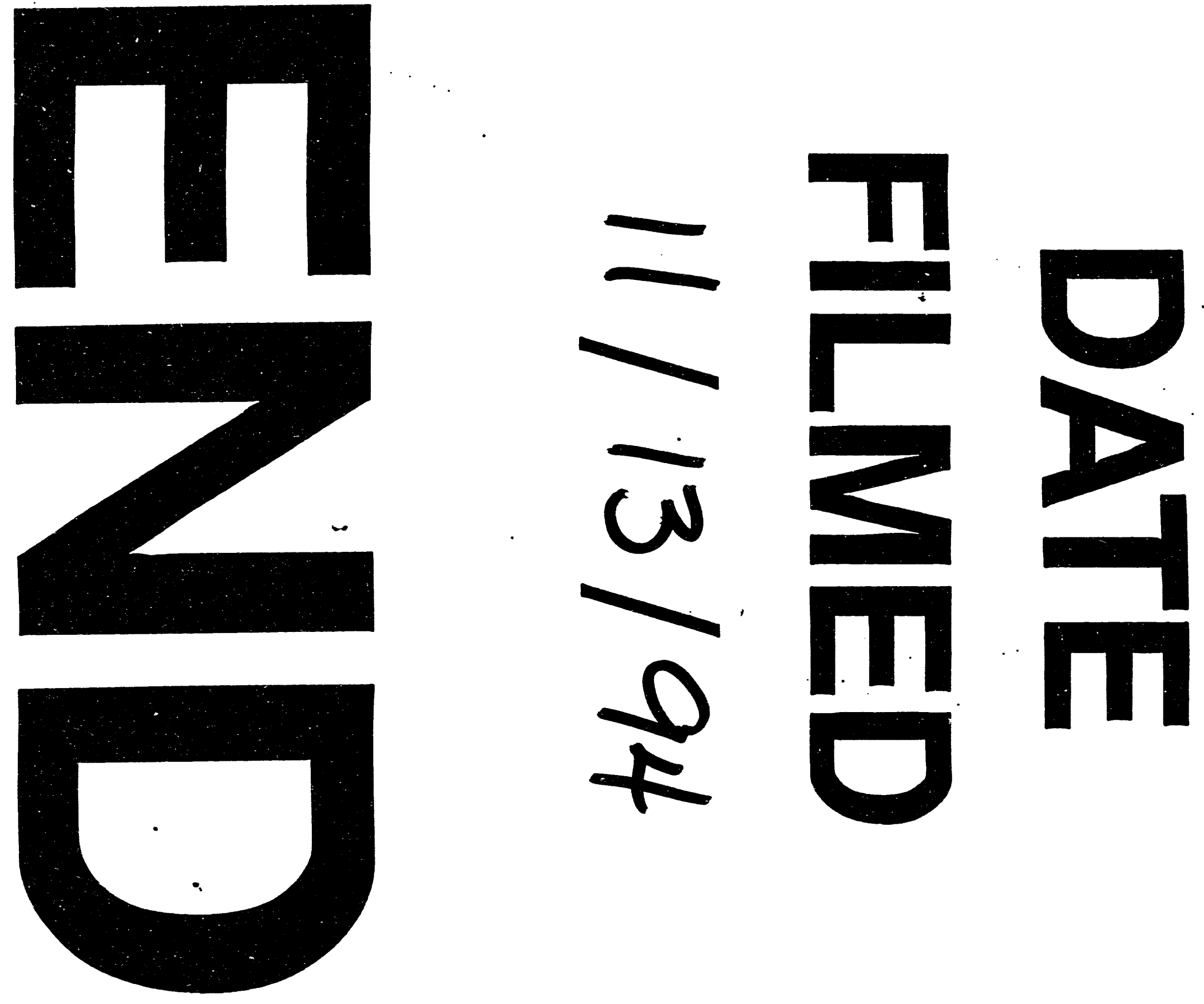